\title{
Immunostimulatory siRNA with a uridine bulge leads to potent inhibition of HBV and activation of innate immunity
}

Tingyu Lan ${ }^{1,3 \dagger}$, Zhiqiang Wei ${ }^{2,4 \dagger}$, Yulin $\mathrm{He}^{2,4 \dagger}$, Song Wan ${ }^{3,4}$, Li Liü ${ }^{3,4}$, Bin Cheng ${ }^{2,4}$, Ruimin Li ${ }^{2,4}$, Hongxia Chen ${ }^{2,4}$, Guohua Liu ${ }^{2,4}$ and Zhongji Meng 1,2,3,4,5*

\begin{abstract}
Background: Hepatitis B virus (HBV) infection is difficult to cure. HBV-specific immune tolerance plays a key role in HBV persistence, and enhancing cellular and humoral immunity will improve the control of HBV infection. The purpose of the study was to explore the anti-HBV and immunostimulatory effects of msiRNAs that introduce unpaired uridine bulges in the passenger strand.

Methods: msiRNAs targeting the HBV S and X genes were designed and named msiHBs and msiHBx, respectively. HepG2 cells were cotransfected with siRNA or msiRNA and the HBV replication-competent plasmid pHY106-wta or pHY106-X15. HepG2.215 cells were transfected with siRNA or msiRNA. The levels of HBsAg, HBeAg, and the cytokines TNF- $\alpha$, IFN- $\alpha$, IFN- $\beta$, IL-1 $\alpha$, and IL-6 in the culture supernatant was detected by ELISA. The levels of intracellular HBV RNA, nuclear HBV replication intermediates, and HBV DNA in the supernatant were measured by quantitative RT-PCR and PCR. The levels of HBV replication intermediates were detected by Southern blotting. Peripheral blood mononuclear cells were transfected with siRNA or msiRNA, and the levels of secreted cytokines IFN- $\alpha$ and IFN- $\beta$ were detected by ELISA. The bioactivity of type I interferons in the supernatants was detected by the virus protection assay.

Results: msiHBx treatment led to a significant decrease in HBsAg (to a negative level) and HBV DNA (95.5\%) in the supernatant and intrahepatocellular HBV replication intermediates (89.8\%) in HepG2 cells with transient HBV replication and in HepG2.2.15 cells. There was no significant difference between msiHBx and siHBx in terms of the reduction in HBV proteins and HBV replication ( $P>0.05$ ). Compared with siHBX, msiHBx treatment of HepG2 cells transfected with the HBV replication-competent plasmid led to a significant increase in the levels of the antiviral cytokines TNF- $a$ (3.3-fold), IFN-a (1.4-fold), and IFN- $\beta$ (2.5-fold) ( $<<0.01$ ), without upregulation of the proinflammatory cytokines IL-1a and IL-6. The virus protection assay results showed msiHBx-mediated type I interferons effectively protected L929 cells against ECMV infection.
\end{abstract}

Conclusions: msiHBx could effectively inhibit HBV expression and replication and induce an antiviral innate immune response without proinflammatory activation. The dual RNAi and immunostimulatory activity of msiRNAs may play an important role in the control of HBV infection.

Keywords: Hepatitis B virus, RNAi, microRNA-like siRNA, msiRNA, Immunostimulation, Innate immunity

*Correspondence: zhongji.meng@163.com

†Tingyu Lan, Zhiqiang Wei and Yulin He contributed equally to this work.

${ }^{2}$ Institute of Biomedical Research, Taihe Hospital, Hubei University

of Medicine, No. 32, South Renmin Road, Shiyan 442000, Hubei Province, China

Full list of author information is available at the end of the article

\section{Background}

Chronic hepatitis B (CHB), a disease caused by hepatitis $B$ virus (HBV), is a global public health problem and the main cause of liver failure, liver cirrhosis, and hepatocellular carcinoma [1]. Currently, pegylated interferon- $\alpha$ 
(PEG-IFN- $\alpha$ ) and nucleos(t)ide analogs (NAs) are the main drug therapies for CHB. PEG-IFN- $\alpha$ is effective only in a small number of CHB patients and is limited by its high cost and severe adverse effects. NAs, especially entecavir, tenofovir, and tenofovir alafenamide fumarate (TAF), are convenient to take orally and are widely used in the treatment of CHB; however, NAs cannot completely eliminate $\mathrm{HBV}$ and need long-term application, which may cause problems such as drug resistance [2]. Thus, it is urgent to develop more effective agents to pursue a cure for HBV infection.

It is generally accepted that high levels of HBV viral loads, hepatitis B surface antigen (HBsAg) and hepatitis $\mathrm{B}$ e antigen (HBeAg) lead to HBV-specific immune tolerance in patients with chronic HBV infection [3]. Persistent HBV infection causes the dysfunction and exhaustion of $\mathrm{T}$ cells and the downregulation and dysfunction of innate immunity-related factors/pathways, especially the Toll-like receptor (TLR) pathway. TLRs are widely expressed in immune cells, hepatocytes, and nonparenchymal liver cells (NPCs) [4,5]. The activation of TLRs in the liver is essential for the establishment of antiviral homeostasis. The reduction in TLR expression caused by chronic HBV infection results in HBV escape from immune cell surveillance, thereby maintaining HBV-specific immune tolerance [6]. Reducing circulating and intrahepatic HBV particles and proteins is a prerequisite for rebuilding/restoring an effective HBV-specific immune response [3].

RNA interference (RNAi) technology can specifically degrade target RNA and has shown potential curative effects in anti-HBV research by significantly reducing HBsAg levels and promoting the elimination of HBsAg. A variety of small interfering RNA (siRNA)-based drugs targeting HBV sequences are currently undergoing assessment in phase I and II clinical trials and have shown promising prospects [7-9]. RNAi is an evolutionarily conserved antiviral mechanism that relies on siRNA of 21-23 base-pair-long double-stranded RNA. Interestingly, previous studies reported that some siRNAs can stimulate the innate immune response in a sequencespecific manner through the activation of retinoic acid-inducible gene-I (RIG-I)/melanoma differentiationassociated protein 5 (MDA5), protein kinase receptor (PKR), and the TLR-3 and TLR-7/8 signaling pathways [10-12]. These siRNAs are called immunostimulatory siRNAs (isiRNAs) which can degrade their target RNA to knock down the expression of the target protein on the one hand and enhance the innate immune response on the other hand. The dual function of isiRNAs may play an important role in antiviral and antitumor therapy [13].

Various isiRNAs have been tested in anti-HBV research $[14,15]$. In HBV transgenic mice, tail injection of guanidinopropyl (GP)-modified siRNA resulted in a decrease in HBsAg of approximately $80 \%$ that lasted for a long time (2 weeks). Toxicity or induction of the proinflammatory factors consisting of interleukin (IL)-6, IFN$\gamma$, and tumor necrosis factor (TNF)- $\alpha$ was not observed after injection of the GP-modified siRNAs [16]. The addition of a $5^{\prime}$-triphosphate (3p) to the siRNAs generated ligands for RIG-I. A $5^{\prime}$-end triphosphate siRNA (3p-siRNA) could induce a RIG-I-dependent antiviral type I IFN response in HBV-infected primary human hepatocytes and HepG2.2.15 cells. In addition, 3p-siRNAs showed more pronounced and long-term suppression of HBV DNA replication and mRNA transcription than normal siRNAs targeting the same sequences [17].

This study introduced a microRNA (miRNA)-like unpaired uridine bulge in the passenger strand of siRNAs to design novel miRNA-like siRNAs (msiRNAs) targeting the HBV sequence and explored its role in the inhibition of HBV gene expression and replication and the activation of innate immune responses.

\section{Methods \\ siRNAs and plasmids}

The sequences of siHBs and siHBx were obtained from a previous study [18]. msiRNAs were generated by substituting the 9-12th nucleotides of the passenger strand of siRNAs with unpaired uracil nucleotides (Fig. 1). msiRNAs targeting the HBV S and X genes with the same target sequences as siHBs and siHBx were designed and named msiHBs and msiHBx, respectively. siNC was purchased from Sangon Biotech (Shanghai, China) and used as a negative control. All siRNAs were synthesized by Sangon Biotech (Shanghai, China). The HBV replication-competent plasmid pHY106-wta (1.3 copies of HBV generated from pSM2 [19]) was a kind gift from Prof. Mengji Lu at Essen University Hospital, Germany. The HBV replication-competent plasmid pHY106-X15 was constructed by cloning a clinically derived single copy of the HBV sequence (genotype C, GenBank accession No. KM213037) into the pHY106 backbone.

\section{Cell culture and transfection}

HepG2.2.15 cells, HepG2 cells, and Huh7 cells were cultured at $37{ }^{\circ} \mathrm{C}$ in a $\mathrm{CO}_{2}$ incubator. HepG2.2.15 cells were routinely cultured in RPMI-1640 medium (Gibco, America) supplemented with $10 \%$ inactivated fetal bovine serum (FBS), $100 \mathrm{U} / \mathrm{mL}$ penicillin, $100 \mu \mathrm{g} / \mathrm{mL}$ streptomycin (Gibco, America), $1 \%$ nonessential amino acids (NEAA), $1 \%$ HEPES, and $500 \mu \mathrm{g} / \mathrm{mL}$ G418 (Gibco, America). HepG2 cells and Huh7 cells were cultured in DMEM medium, supplemented with $10 \%$ inactivated FBS, $100 \mathrm{U} /$ $\mathrm{mL}$ penicillin, $100 \mu \mathrm{g} / \mathrm{mL}$ streptomycin (Gibco, America), $1 \%$ NEAA, and $1 \%$ HEPES. The transfection of siRNAs 
siHBs 5' GAAUCCUCACAAUACCGCAtt

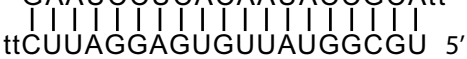

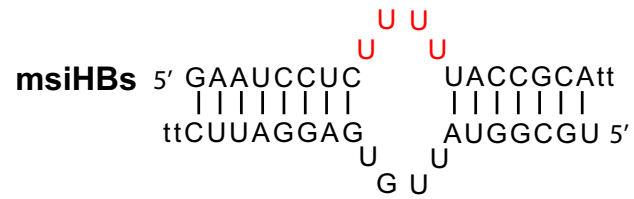

SiHBx 5' GAGGACUCUUGGACUCUCAtt I I I I I I I I I I I I I I I I I I I I
ttCUCCUGAGAACCUGAGAGU 5'

\author{
msiHBx 5' GAgGACUCUU ACUCUCAtt \\ IIIIIIII IIIIIII \\ ttCUCCUGAGAA UGAGAGU 5'
}

Fig. 1 The sequences and structures of siRNAs and msiRNAs with unpaired uracil bulges. The uracil substitution at the 9-12th nucleotides of the passenger strand of siRNAs is indicated in red. siHBs, siRNA targeting the HBV S gene. siHBx, siRNA targeting the HBV X gene. msiHBs, msiRNA targeting the HBV S gene. msiHBX, msiRNA targeting the HBV X gene. siNC was purchased from Sangon Biotech (Shanghai, China) and used as a negative control

and plasmids was performed with Lipofectamine 2000 transfection reagent (Invitrogen, America) according to the manufacturer's instructions. Peripheral blood mononuclear cells (PBMCs) from CHB patients (obtained with signed informed consent) were separated by Ficoll density gradient centrifugation and cultured in RPMI1640 + L-glutamine medium (Gibco, America) supplemented with $10 \%$ inactivated FBS. DOTAP liposomal transfection reagent (Sigma-Aldrich) was used in the transfection of PBMCs with siRNAs.

\section{PCR and reverse transcription (RT) PCR assays}

Total DNA in the supernatant was extracted with a TIAN amp Virus DNA/RNA Kit (Tiangen, China), and $2 \mu \mathrm{l}$ of the extracted DNA solution was subjected to real-time PCR to quantify extracellular HBV virions. HBV replication intermediates in cells were extracted according to a previous study [20], and $2 \mu \mathrm{l}$ of the extracted DNA solution was subjected to real-time PCR. Real-time PCR was carried out by using TB Green Premix Ex Taq II (TaKaRa) in a Step-OnePlus Real-Time PCR System (Thermo Fisher Scientific) using the primers $5^{\prime}$-GTT GCCCGTTTGTCCTCTAATTC and 5'-GGAGGGATA CATAGAGGTTCCTT. PCR was performed with the following parameters for 40 cycles: $95{ }^{\circ} \mathrm{C}$ for $5 \mathrm{~s}$ and $60{ }^{\circ} \mathrm{C}$ for $30 \mathrm{~s}$.

Total RNA was purified from cells using TRIzol reagent (Invitrogen) according to the manufacturer's instructions. One-step real-time RT-PCR was performed with $100 \mathrm{ng}$ of total RNA using a One-step RT-PCR kit (TaKaRa) on a Step-One Plus Real-Time PCR System (Thermo Fisher Scientific) using the primers $5^{\prime}$-CCGTCTGTGCCT TCTCATCT and 5'-TAATCTCCTCCCCCAACTCC to detect HBV RNA levels. RT-PCR was performed with the following parameters for 40 cycles: $95{ }^{\circ} \mathrm{C}$ for $30 \mathrm{~s}, 55^{\circ} \mathrm{C}$ for $30 \mathrm{~s}$, and $72{ }^{\circ} \mathrm{C}$ for $30 \mathrm{~s}$.

\section{ELISA}

HBsAg and HBeAg in the culture supernatants were detected by the HBsAg ELISA Kit and HBeAg ELISA Kit (Kehua Bio engineering, China). The levels of IL-1 $\alpha$, IL- 6 , IFN- $\alpha$, IFN- $\beta$, and TNF- $\alpha$ in the culture supernatants were quantified by ELISA kits (BD Biosciences).

\section{Southern blotting}

The intracellular HBV replication intermediates extracted above were detected by Southern blotting using DIG high prime DNA labeling and detection starter kit II (Roche).

\section{Virus protection assay}

The bioactivity of type I IFNs in supernatants was analyzed by the virus protection assay as described elsewhere [21]. Briefly, L929 cells were seeded into 96-well plates and cultured in $100 \mu \mathrm{l}$ RPMI-1640 medium supplemented with $10 \% \mathrm{FBS}$ at $37^{\circ} \mathrm{C}$ and $5 \% \mathrm{CO}_{2}$ until they reached $100 \%$ confluence. After the culture medium was discarded, $100 \mu \mathrm{l}$ of RPMI-1640 medium with 1:2 to 1:256 dilutions of the supernatants of siRNA-treated HepG2 cells was added to the L929 cells for an additional incubation of $24 \mathrm{~h}$. Then, murine encephalomyocarditis virus (EMCV) was added to the cells and incubated for another $24 \mathrm{~h}$. The cells were stained and fixed with $0.1 \%$ crystal violet in $20 \%$ ethanol. A unit of IFN was defined by its ability to protect $50 \%$ of the cells from cell death.

\section{Statistical analyses}

The results of measurement data are expressed as the means \pm SEM. Statistical analyses were performed using GraphPad Prism software version 7 (La Jolla, CA, USA). Differences between groups were assessed with twotailed Student's $t$ test of variance (ANOVA). $\mathrm{P}<0.05$ was considered statistically significant. All experiments were repeated independently at least three times. 


\section{Results}

msiRNA ( $m s i H B x$ ) with unpaired uracil bulges showed similar effects as standard siRNA (siHBx) in knocking down HBsAg

To investigate the knockdown effects of msiRNAs, various concentrations of msiHBs, msiHBx, siHBs, or siHBx were cotransfected with the HBV replicationcompetent plasmid pHY106-wta or pHY106-X15 into HepG2 cells. The ELISA results showed that the siRNAs knocked down the levels of HBsAg in the supernatants in a dose-dependent manner (Fig. 2). The levels of HBsAg decreased significantly even in the presence of very low concentrations $(0.2 \mathrm{nM})$ of msiRNAs or siRNAs and to a level of less than $5 \%$ of untreated controls in the presence of $20 \mathrm{nM}$ msiRNAs or siRNAs. Compared with the standard siRNAs (siHBs and siHBx), msiHBs was much less effective than siHBs, while msiHBx displayed a similar or even better inhibitory effect than siHBx on HBsAg production; therefore, msiHBx was used in subsequent experiments.

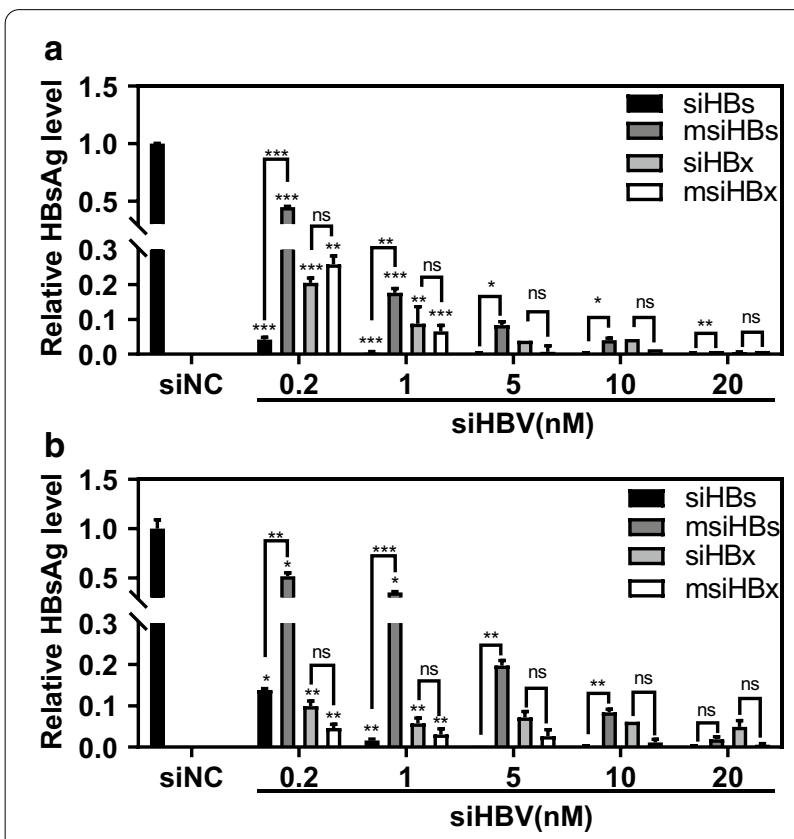

Fig. 2 Comparison of siRNAs and msiRNAs in their ability to knock down HBsAg expression. HepG2 cells were cotransfected with $1 \mu \mathrm{g}$ of the HBV replication-competent plasmid pHY106-wta (a) or pHY106-X15 (b) and msiHBs, msiHBx, siHBs, or siHBx at various concentrations or with $20 \mathrm{nM}$ siNC as a negative control. The levels of HBsAg in supernatants were detected by ELISA at $72 \mathrm{~h}$ after transfection. ns, no significant difference. ${ }^{*} p<0.05$. ${ }^{* *} p<0.01$. ${ }^{* * *} p<0.001$. siNC was purchased from Sangon Biotech (Shanghai, China) and used as a negative control. siHBs, siRNA targeting the HBV S gene. siHBx, siRNA targeting the HBV X gene. msiHBs, msiRNA targeting the HBV $S$ gene. msiHBx, msiRNA targeting the HBV X gene
msiHBx significantly inhibited the replication and gene expression of HBV in cells with transient or stable HBV replication

To further verify the inhibitory effects of msiHBx on HBV replication and gene expression, Huh7 cells were cotransfected with siHBx or msiHBx and the HBV replication-competent plasmid pHY106-wta. At $72 \mathrm{~h}$ after transfection, the levels of $\mathrm{HBsAg}$ and $\mathrm{HBeAg}$ in the supernatants (Fig. 3a), intracellular HBV RNA (Fig. 3c), and HBV DNA in the supernatants (Fig. 3d) decreased significantly to a level less than $5 \%$ of those treated with siRNA negative control (siNC). Compared with the siNC treatment, both siHBx and msiHBx treatment led to a significant decrease in HBV replication intermediates by over $90 \%$ (Fig. 3b, e). There was no significant difference between siHBx and msiHBx in the RNAi effects on HBV replication and gene expression $(\mathrm{P}>0.05)$.

In HBV stably producing HepG2.2.15 cells transfected with siHBx or msiHBx, the levels of HBsAg and HBeAg in the supernatants (Fig. 3f) and intracellular HBV replication intermediates (Fig. 3h) were decreased to less than $60 \%$ compared with those in cells transfected with siNC. The reduction in HBV replication intermediates was also verified by Southern blotting (Fig. 3g). Again, there was no significant difference between siHBx and msiHBx in the inhibitory effects on HBV replication and gene expression in the HBV stably producing HepG2.2.15 cells $(\mathrm{P}>0.05)$.

\section{msiHBx with a uridine-bulge modification enhanced antiviral cytokine secretion}

To explore whether msiHBx with an unpaired miRNAlike uridine-bulge modification could stimulate the innate immune response, HepG2 cells were transfected with $1 \mathrm{nM}$ or $20 \mathrm{nM}$ siHBx, msiHBx, or siNC alone as a control or in combination with the HBV replication-competent plasmid pHY106-X15. The results showed that in the absence of HBV, there was no significant difference in the levels of IFN- $\alpha$, IFN- $\beta$, TNF- $\alpha$, IL- $1 \alpha$, and IL- 6 cytokine production in the supernatants of HepG2 cells treated with siHBx, msiHBx, or siNC. When msiHBx was cotransfected with the HBV replication-competent plasmid pHY106-X15 into HepG2 cells, a significant increase in the levels of the antiviral cytokines IFN- $\alpha$ (2.4-fold), IFN- $\beta$ (3.3-fold), and TNF- $\alpha$ (2.4-fold) was induced compared with those in siNC-treated cells (Fig. $4 \mathrm{a}-\mathrm{c}$ ), but there was no significant increase in the levels of the inflammatory cytokines IL-1 $\alpha$ and IL-6 (P > 0.05) (Fig. 4d, e). In contrast, in HepG2 cells cotransfected with siHBx and the HBV replication-competent plasmid pHY106$\mathrm{X} 15$, a significant increase in the levels of IL-1 $\alpha$ and IL-6 was observed $(\mathrm{P}<0.01)$, and the levels of antiviral 


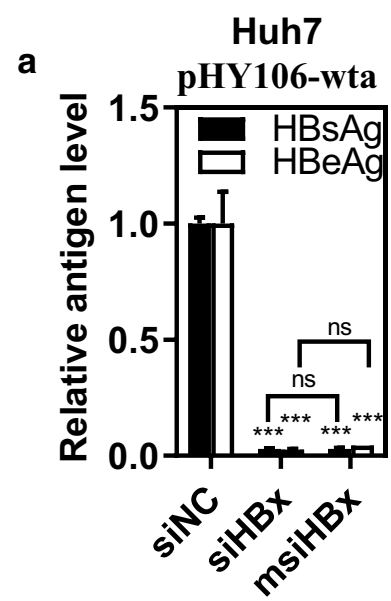

d

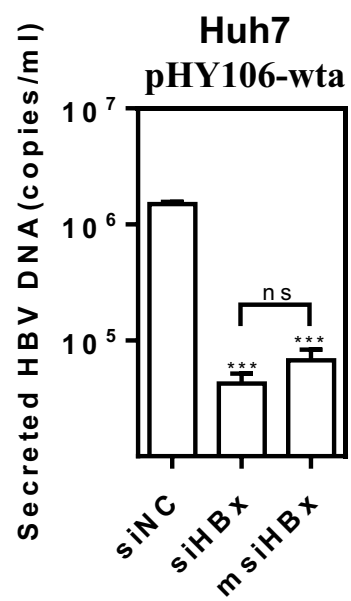

$\mathbf{f}$

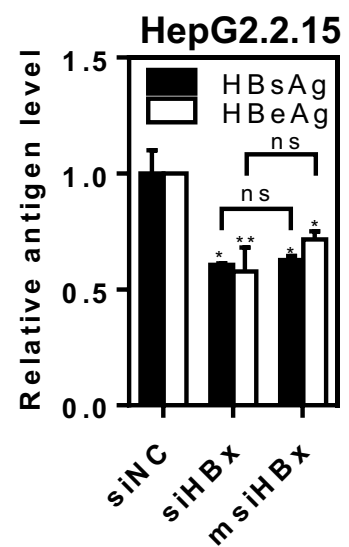

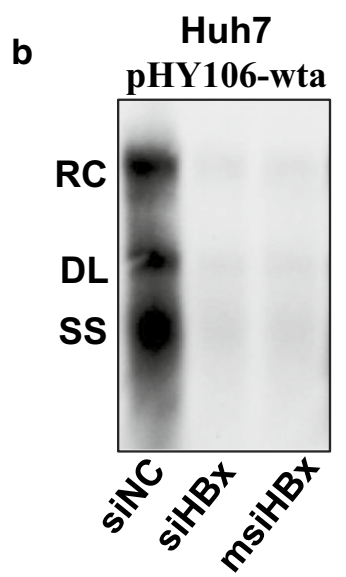

c

Huh7

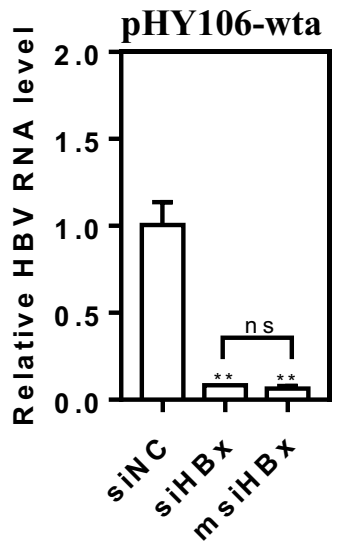

Huh7

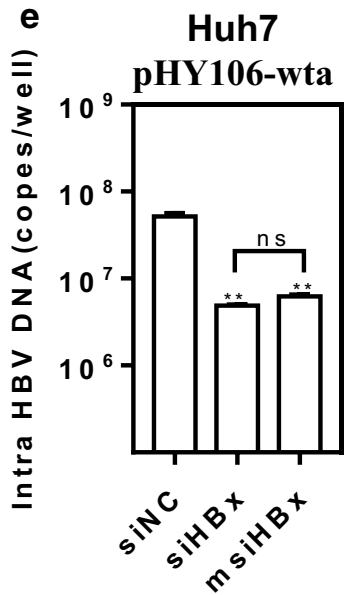

g

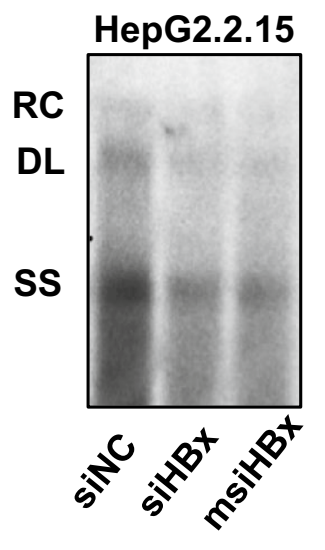

h

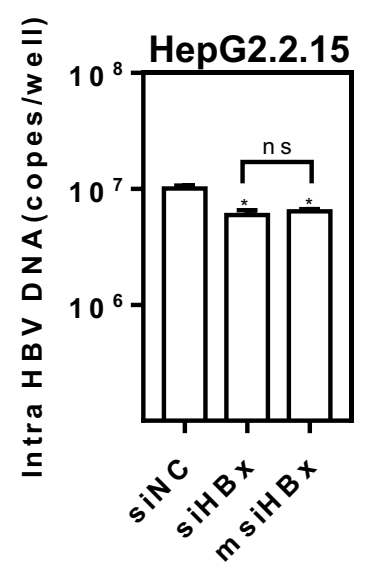

Fig. 3 Inhibitory effects of msiHBx on HBV replication and gene expression. Huh7 cells were cotransfected with $1 \mu \mathrm{gg}$ of pHY106-wta and $20 \mathrm{nM}$ msiHBx, siHBx, or siNC as a control. The levels of HBsAg and HBeAg in cell culture supernatants (a), HBV replicative intermediates (b, e), HBV DNA in cell culture supernatants (d), and HBV RNA in cells (c) were analyzed at $72 \mathrm{~h}$ after transfection by ELISA, Southern blotting, PCR, and One-Step RT-PCR, respectively. HepG2.2.15 cells were transfected with $20 \mathrm{nM} \mathrm{msiHBx}$, siHBx, or siNC as a control. The HBsAg and HBeAg levels in cell culture supernatants $(\mathbf{f})$ and HBV replicative intermediates $(\mathbf{g}, \mathbf{h})$ were analyzed at $72 \mathrm{~h}$ after transfection by ELISA, Southern blotting, and PCR, respectively. ns, no significant difference. ${ }^{*} p<0.05 .{ }^{* *} p<0.01 .{ }^{* * *} p<0.001$. siNC was purchased from Sangon Biotech (Shanghai, China) and used as a negative control. siHBX, siRNA targeting the HBV X gene. msiHBX, msiRNA targeting the HBV X gene. RC, relaxed circular DNA. DL, double-stranded linear DNA. SS, single-stranded DNA 
a

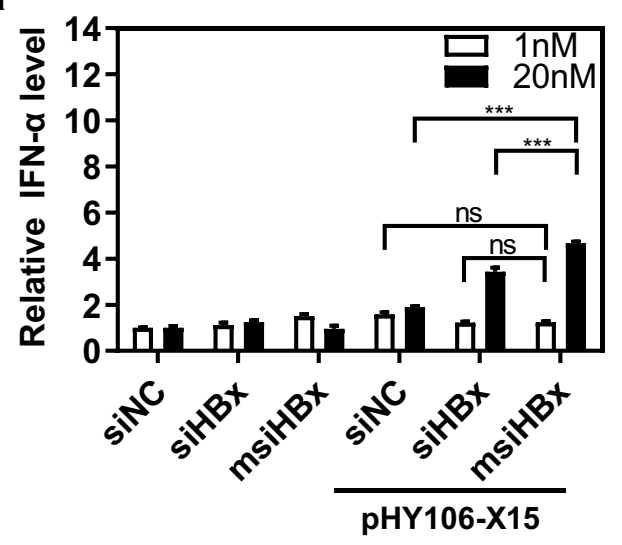

C

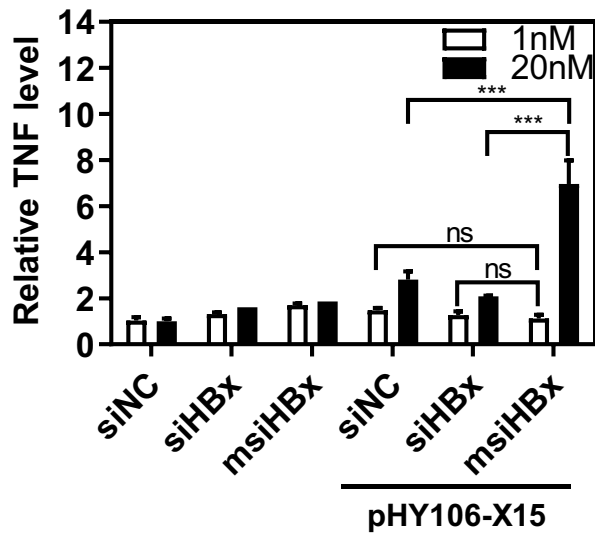

d

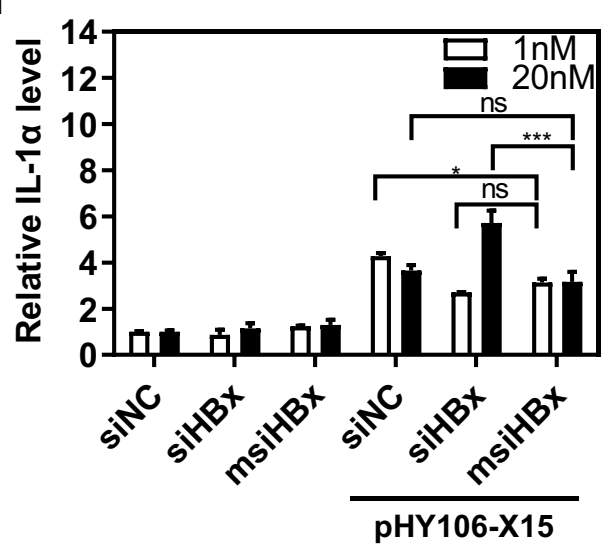

b

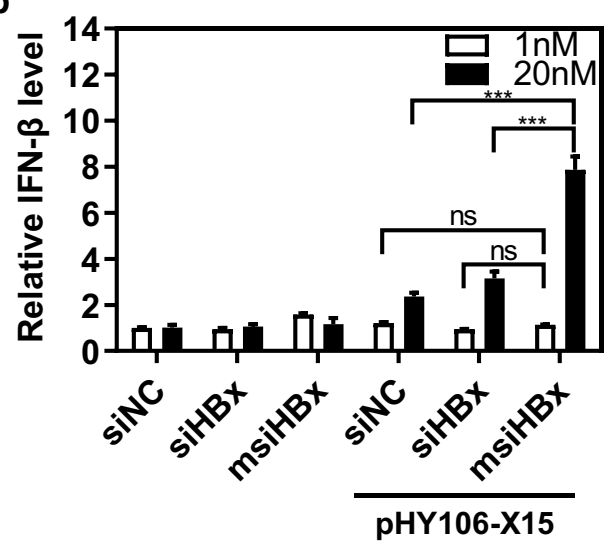

e

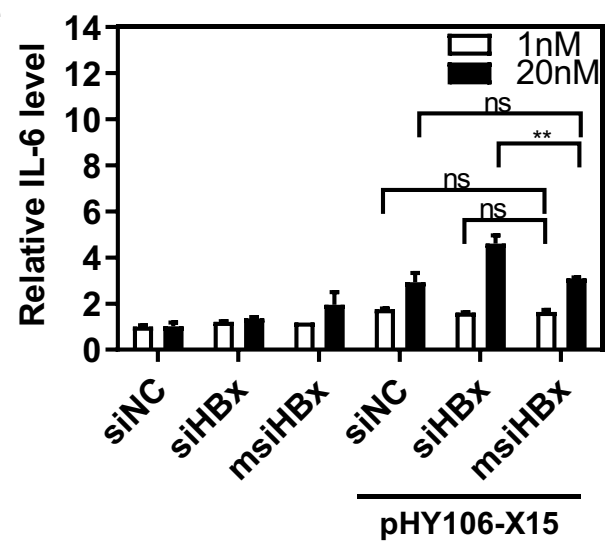

Fig. 4 Enhanced antiviral cytokine secretion by msiHBx-directed HBV silencing. HepG2 cells were transfected with msiHBx, siHBx, or siNC alone or cotransfected with $1 \mu \mathrm{g}$ of the HBV replication-competent plasmid pHY106-X15. The levels of IFN-a (a), IFN- $\beta$ (b), TNF (c), IL-1a (d), and IL-6 (e) in supernatants were detected at $72 \mathrm{~h}$ after transfection by ELISA. ns, no significant difference. ${ }^{*} p<0.05 .{ }^{* *} p<0.01 .{ }^{* * *} p<0.001$. siNC was purchased from Sangon Biotech (Shanghai, China) and used as a negative control. siHBX, siRNA targeting the HBV X gene. msiHBX, msiRNA targeting the HBV X gene

cytokines IFN- $\alpha$ and IFN- $\beta$ increased to a lesser extent compared with those induced by msiHBx treatment.

Further, when msiHBx or siHBx was transfected into PBMCs isolated from $\mathrm{CHB}$ patients, ELISA results showed that both msiHBx and siHBx caused a significant increase in the production of IFN- $\alpha$ and IFN- $\beta$, and msiHBx induced more IFN- $\alpha$ (fivefold $v s$. twofold, 
$\mathrm{P}<0.01)$ and IFN- $\beta$ (4.5-fold $v$ s. 2.4 -fold, $\mathrm{P}<0.01$ ) than siHBx (Fig. 5a, b).

\section{Type I interferons induced by msiHBx protected L929 cells from EMCV infection}

In the virus protection assay, EMCV infection caused L929 cell death. However, in the presence of supernatants from HepG2 cells cotransfected with the HBV replication-competent plasmid pHY106-X15 and msiHBx or siHBx, the viability of L929 cells was significantly improved $(\mathrm{P}<0.001)$, especially in the presence of supernatants from HepG2 cells cotransfected with pHY106-X15 and msiHBx $(\mathrm{P}<0.001)$ (Fig. 5c), which indicated that type I interferons induced by siHBx- and
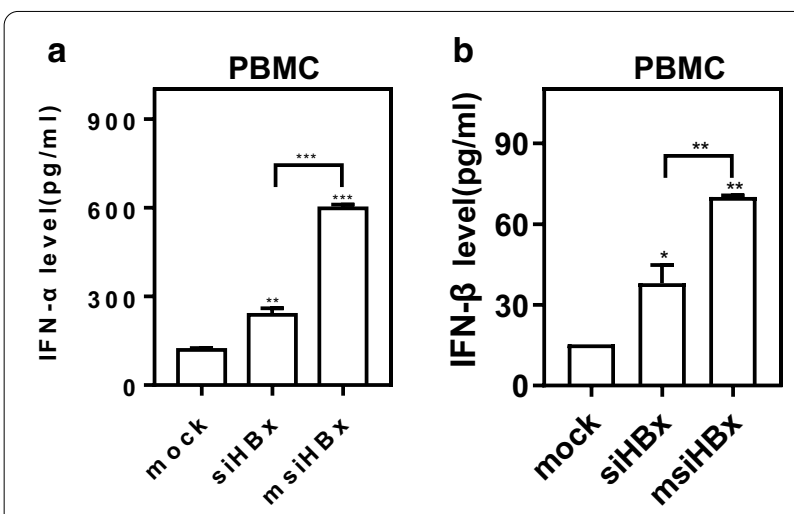

C

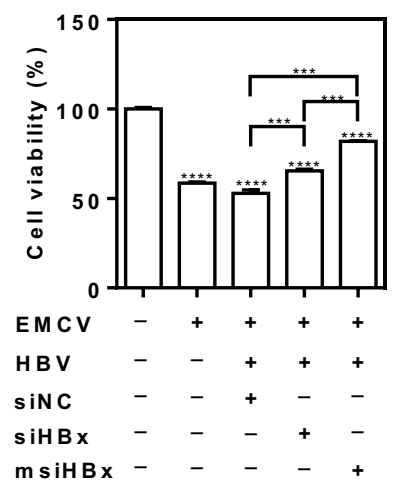

Fig. 5 Type I interferons induced by msiHBx protected L929 cells from EMCV infection. PBMCs were transfected with siHBx or msiHBx at concentrations of $20 \mathrm{nM}$. The levels of IFN- $a(\mathbf{a})$ and IFN- $\beta$ (b) in supernatants were detected at $72 \mathrm{~h}$ by ELISA. The bioactivity of type I IFNs in cell culture supernatants from HepG2 cells cotransfected with $1 \mu \mathrm{g}$ of $\mathrm{pHY} 106-\mathrm{X} 15$ and siHBx or msiHBx was determined by the virus protection assay $(\mathbf{c})$. ns, no significant difference. ${ }^{*} p<0.05 .{ }^{* *} p<0.01 .{ }^{* * *} p<0.001$. siNC was purchased from Sangon Biotech (Shanghai, China) and used as a negative control. siHBx, siRNA targeting the HBV X gene. msiHBx, msiRNA targeting the $\mathrm{HBV} X$ gene. PBMCs, peripheral blood mononuclear cells. EMCV, encephalomyocarditis virus
msiHBx-directed RNAi protected L929 cells from EMCV infection.

\section{Discussion}

In this study, in an HBV transient replication-competent system (HepG2 cells transfected with the HBV replication-competent plasmid pHY106-wta or pHY106-X15) and HBV stably producing HepG2.2.15 cells, the msiRNA msiHBx with a uridine-bulge modification significantly decreased the levels of HBV mRNA, the expression of $\mathrm{HBsAg}$ and $\mathrm{HBeAg}, \mathrm{HBV}$ replication intermediates, and HBV virions. msiHBx showed similar or even better RNAi effects on HBV than the traditional siRNA siHBx targeting the same sequence, indicating that the RNAi activity of msiHBx was not affected by the uridine-bulge modification. On the other hand, msiHBx upregulated the expression of the antiviral cytokines IFN- $\alpha$ and IFN- $\beta$ directly (msiHBx transfected into PBMCs) or indirectly by RNAi-directed immune stimulation (msiHBx cotransfected with the HBV replication-competent plasmid pHY106-wta or pHY106-X15 in HepG2 cells). The antiviral ability of type I interferons was confirmed in a viral protection assay, and msiHBx did not stimulate cells to secrete the inflammatory cytokines IL- $1 \alpha$ or IL- 6 , suggesting that the msiRNA could activate cellular antiviral innate immunity without inducing an inflammatory response. In contrast, the HBV RNAi effects mediated by the traditional siRNA siHBx also caused the upregulated expression of the antiviral cytokines IFN- $\alpha$ and IFN- $\beta$, which was consistent with a previous study [13]. However, siHBx did not induce TNF- $\alpha$ but upregulated the inflammatory cytokines IL-1 $\alpha$ and IL-6. In this study, compared with the traditional siHBx, msiHBx exhibited an enhanced ability to upregulate type I interferons without obvious inflammatory stimulation, which is beneficial for HBV immune control.

HBsAg and HBeAg play an important role in HBVspecific immune tolerance and persistent infection [6]. $\mathrm{HBeAg}$ loss/seroconversion is indicative of reduced virus replication, which is one of the goals of antiviral treatments. The consistent decrease in HBsAg facilitates the recovery of the $\mathrm{HBV}$-specific immune response, which is beneficial for the immune control of HBV infection. "A functional cure", which is characterized by HBsAg loss and/or HBsAg seroconversion, is the ideal treatment goal for HBV infection [15]. RNAi, with the ability to degrade mRNA in a sequence-specific manner, shows unique capabilities in reducing $\mathrm{HBsAg}$ and $\mathrm{HBeAg}[9,22$, 23]. Preclinical and clinical studies have also confirmed that siRNA-based treatment could significantly reduce the levels of $\mathrm{HBsAg}$ and $\mathrm{HBeAg}$ in vivo and improve host immune function [24]. siRNA targeting the $S$ gene (siHBs) can act on the 2.4-kb and 2.1-kb HBV mRNA to 
reduce $\mathrm{HBsAg}$, preS2, and preS1 protein levels and act on the 3.5-kb HBV pregenomic RNA (pgRNA), leading to the degradation of $\mathrm{HBV}$ pgRNA and reduction in $\mathrm{HBcAg}$ and $\mathrm{HBeAg}$, altogether resulting in the inhibition of $\mathrm{HBV}$ replication and gene expression. siRNA targeting the $\mathrm{X}$ gene (siHBx) can act on all HBV mRNAs. Therefore, in addition to the above effects, siHBx also degrades the 0.7-kb HBx mRNA, thereby downregulating the expression of $\mathrm{HBxAg}$. Since HBxAg plays an important role in maintaining the stability of HBV covalently closed circular DNA (cccDNA), inhibition or mutation of HBxAg can decrease the level of cccDNA [25]. Therefore, siHBx can theoretically exert a better anti-HBV effect than siHBs; thus, the siHBx targeting the $\mathrm{X}$ gene was selected for further investigation in this study.

The presence of uridine in RNA induces TLR7/8dependent cytokine production, which correlates with overall uridine moieties [13, 26, 27]. The insertion of unpaired miRNA-like uracil at the 9-12 bases of the siRNA messenger strand will enhance the immunostimulatory ability of the siRNA as the " $U$ " content of the siRNA increases. On the other hand, the miRNAlike stem-loop structure of msiRNA can also enhance the immunostimulatory activity of an siRNA through TLR7/8 [28]. Michael et al. [28] designed a bifunctional siRNA with both immunostimulatory and specific silencing activities. The introduction of a miRNA-like unpaired uridine-bulge modification strongly stimulated human immune cells, leading to the production of cytokines, thereby protecting HeLa cells from Semliki Forest virus infection.

In this study, the RNAi activity of siHBs was significantly affected after the introduction of unpaired uridine-bulge (msiHBs), while the RNAi activity of msiHBx was consistent with or even better than that of siHBx. A deeper look into the secondary structure of the two msiRNAs indicates that the 4 " $U$ " of the 9-12 bases of msiHBs are unpaired, while only $2 / 4$ " $U$ " of the $9-12$ bases of msiHBx are unpaired. Thus, the larger ring-shaped protrusion of msiHBs may affect the binding efficiency or interaction of msiHBs and Dicer, thereby accounting for the reduced RNAi activity.

A significantly increased production of IFN- $\alpha$ and IFN- $\beta$ was induced in PBMCs transfected with $\mathrm{msiHBx}$, further validating the immunostimulatory activity of msi$\mathrm{HBx}$. No obvious cytokines were found to be upregulated in HepG2 cells transfected with msiHBx or siHBx. This is due to defects in the innate immune signaling pathways of hepatocarcinoma cells, which require stronger stimulation signals to activate [6]. Transfection of the traditional siRNA siHBx into PBMCs also induced IFN- $\alpha$ and IFN- $\beta$, suggesting that siHBx itself also possesses certain immunostimulatory activity, but siHBx-mediated IFN- $\alpha$ and IFN- $\beta$ production was much lower than that induced by $\mathrm{msiHBx}$, further confirming that a uracil ring-shaped protrusion modification can enhance the immunostimulatory activity of siRNAs. RNAi-mediated degradation of target mRNA can activate cellular innate immunity through the TLR-7/8 or PKR pathway [6]. In this study, both msiHBx- and siHBx-directed HBV RNAi (msiHBx or siHBx cotransfected with pHY106-wta in HepG2 cells) upregulated the expression of IFN- $\alpha$ and IFN- $\beta$, providing more evidence in favor of RNAi-mediated immune stimulation. Compared with siHBx, msiHBx-directed HBV RNAi induced higher levels of IFN- $\alpha$ and IFN- $\beta$, further indicating that the presence of the uracil ringshaped protrusion structure can enhance the immunostimulatory activity of RNAi. Interestingly, HBV RNAi directed by the traditional siRNA siHBx stimulated the cells to produce the inflammatory cytokines IL- $1 \alpha$ and IL-6, while HBV RNAi directed by the msiRNA msiHBx did not induce IL- $1 \alpha$ or IL- 6 . msiHBx can enhance the antiviral innate immunity of cells without activating the cellular inflammatory response. This characteristic of msiRNAs is more conducive to the immune control of HBV and limits immune-related inflammatory damage. Therefore, msiRNAs can enhance the secretion of antiviral factors without activating the cellular inflammatory response, in addition to inhibiting the replication and gene expression of HBV. This characteristic of msiRNAs may play an important role in the immune control of the virus and may be developed as a new anti-HBV agent.

\section{Conclusions}

In Conclusion, high levels of HBV virions and proteins are key factors for maintaining persistent infection. Effective anti-HBV treatment strategies need to reduce the HBV loads and antigens on the one hand and restore/enhance the anti-HBV immune response on the other hand. Here, the msiRNA modified by the uracil ring-shaped protrusion structure enhanced the immunostimulatory activity of RNAi without inducing cell inflammation beyond the high RNAi activity. The antiviral/immunostimulatory dual activities of msiRNA are more conducive to the control of HBV, and it is unnecessary to consider immune-related inflammatory damage to the liver. The in vivo antiviral and immunostimulatory activities of the msiRNA and the role of immunostimulatory activity in immune control and clearance of HBV deserve further study.

\section{Abbreviations}

CHB: Chronic hepatitis B; HBV: Hepatitis B virus; PEG-IFN-a: Pegylated interferon-a; NAs: Nucleos(t)ide analogs; TAF: Tenofovir alafenamide fumarate; HBsAg: Hepatitis B surface antigen; HBeAg: Hepatitis B e antigen; TLR: Toll-like receptor; NPCs: Nonparenchymal liver cells; RNAi: RNA interference; siRNA: Small interfering RNA; RIG-I: Retinoic acid-inducible gene-l; MDA5: Melanoma 
differentiation-associated protein 5; PKR: Protein kinase receptor; isiRNAs: Immunostimulatory siRNAs; GP: Guanidinopropyl; IL: Interleukin; TNF: Tumor necrosis factor; 3p: Triphosphate; miRNA: MicroRNA; msiRNAs: MiRNA-like siRNAs; FBS: Fetal bovine serum; NEAA: Nonessential amino acids; PBMCs: Peripheral blood mononuclear cells; RT: Reverse transcription; EMCV: Encephalomyocarditis virus; pgRNA: Pregenomic RNA; cccDNA: Covalently closed circularDNA.

\section{Acknowledgements}

Not applicable.

\section{Authors' contributions}

$T L, Z W, Z M$ participated in writing-original draft, writing-review and editing. $T L, Z W, Y H$ performed the research and data analysis. ZW, YH, ZM participated in the research design. SW, LL participated in collect samples. BC, RL, HC, GL helped with experiments and data analysis. ZM supervised the study. All authors approved the final version for publication and agree to be held accountable for all aspects of the work.

\section{Funding}

This work was supported by the Foundation for Innovative Research Groups of Hubei Provincial Natural Science Foundation (2018CFA031), the National Science and Technology Major Project (Grant Nos. 2018ZX10723203 and 2018ZX10302206), the Hubei Province's Outstanding Medical Academic Leader Program, and the Project of Hubei University of Medicine (FDFR201902, 2020XGFYZR05, YC2019030).

\section{Availability of data and materials}

The datasets used and analyzed during the current study are available from the corresponding author on reasonable request.

\section{Ethics approval and consent to participate}

This research was approved by the ethics committee of Shiyan Taihe Hospital Affiliated with Hubei University of Medicine (Serial no. 2020KS039). The informed consent was signed. The remaining blood from the patient's clinical examination was collected. Peripheral blood mononuclear cells were separated and cultured only for siRNA transfection experiments without infringing on the rights and interests of the patients.

\section{Consent for publication}

Not applicable.

\section{Competing interests}

The authors declare that they have no competing interests.

\section{Author details}

${ }^{1}$ Postgraduate Training Basement of Jinzhou Medicical University, Taihe Hospital, Hubei University of Medicine, Shiyan, China. ${ }^{2}$ Institute of Biomedical Research, Taihe Hospital, Hubei University of Medicine, No. 32, South Renmin Road, Shiyan 442000, Hubei Province, China. ${ }^{3}$ Department of Infectious Diseases, Taihe Hospital, Hubei University of Medicine, Shiyan, China. ${ }^{4}$ Hubei Clinical Research Center for Precise Diagnosis and Treatment of Liver Cancer, Taihe Hospital, Hubei University of Medicine, Shiyan 442000, China. ${ }^{5}$ Hubei Key Laboratory of Embryonic Stem Cell Research, Taihe Hospital, Hubei University of Medicine, Shiyan 442000, China.

Received: 3 December 2020 Accepted: 8 February 2021

Published online: 18 February 2021

\section{References}

1. Revill PA, Chisari FV, Block JM, Dandri M, Gehring AJ, Guo H, Hu J, Kramvis A, Lampertico P, Janssen HLA, et al. A global scientific strategy to cure hepatitis B. Lancet Gastroenterol Hepatol. 2019;4:545-58.

2. Ning $Q$, Wu D, Wang GQ, Ren $H$, Gao ZL, Hu P, Han MF, Wang $Y$, Zhang WH, Lu FM, Wang FS. Roadmap to functional cure of chronic hepatitis B: an expert consensus. J Viral Hepat. 2019;26:1146-55.

3. Dembek C, Protzer U, Roggendorf M. Overcoming immune tolerance in chronic hepatitis B by therapeutic vaccination. Curr Opin Virol. 2018;30:58-67.
4. Ma Z, Cao Q, Xiong Y, Zhang E, Lu M. Interaction between Hepatitis B Virus and toll-like receptors: current status and potential therapeutic use for chronic hepatitis B. Vaccines. 2018;6:6.

5. Du K, Liu J, Broering R, Zhang X, Yang D, Dittmer U, Lu M. Recent advances in the discovery and development of TLR ligands as novel therapeutics for chronic HBV and HIV infections. Expert Opin Drug Discov. 2018:13:661-70.

6. Meng Z, Chen Y, Lu M. Advances in Targeting the Innate and Adaptive Immune Systems to Cure Chronic Hepatitis B Virus Infection. Front Immunol. 2019;10:3127.

7. Yuen MF, Schiefke I, Yoon JH, Ahn SH, Heo J, Kim JH, Lik Yuen Chan H, Yoon KT, Klinker H, Manns M, et al. RNA interference therapy with ARC520 results in prolonged hepatitis B surface antigen response in patients with chronic hepatitis B infection. Hepatology. 2020;72:19-31.

8. Wooddell C, Zhu R, Hamilton H, Chu Q, Sternard H, Schumacher J, Schluep T, Seefeld M, Li Z, Given B. Development of subcutaneously administered RNAi therapeutic ARO-HBV for chronic hepatitis B virus infection. J Hepatol. 2018;68:S18-9.

9. Lee ACH, Heyes J, Ye X, Holland R, Thi EP, Wood M, Judge A, Snead NM, Martin A, Sofia MJ. Durable inhibition of hepatitis B virus replication and antigenemia using a subcutaneously administered siRNA agent in preclinical models. J Hepatol. 2018;68:S18.

10. Heil F, Hemmi H, Hochrein H, Ampenberger F, Kirschning C, Akira S, Lipford G, Wagner H, Bauer S. Species-specific recognition of single-stranded RNA via toll-like receptor 7 and 8. Science. 2004;303:1526-9.

11. Gantier MP, Tong S, Behlke MA, Xu D, Phipps S, Foster PS, Williams BR. TLR7 is involved in sequence-specific sensing of single-stranded RNAs in human macrophages. J Immunol. 2008;180:2117-24.

12. Diebold SS, Massacrier C, Akira S, Paturel C, Morel Y. Reis e Sousa C: nucleic acid agonists for Toll-like receptor 7 are defined by the presence of uridine ribonucleotides. Eur J Immunol. 2006;36:3256-67.

13. Meng Z, Lu M. RNA interference-induced innate immunity, off-target effect, or immune adjuvant? Front Immunol. 2017:8:331.

14. Das S, Ramakrishnan K, Behera SK, Ganesapandian M, Xavier AS, Selvarajan S. Hepatitis B Vaccine and Immunoglobulin: key concepts. J Clin Transl Hepatol. 2019;7:165-71.

15. van den Berg F, Limani SW, Mnyandu N, Maepa MB, Ely A, Arbuthnot P. Advances with RNAi-based therapy for hepatitis B virus infection. Viruses. 2020;12:851.

16. Marimani MD, Ely A, Buff MC, Bernhardt S, Engels JW, Scherman D, Escriou $V$, Arbuthnot P. Inhibition of replication of hepatitis B virus in transgenic mice following administration of hepatotropic lipoplexes containing guanidinopropyl-modified siRNAs. J Control Release. 2015;209:198-206.

17. Chen X, Qian Y, Yan F, Tu J, Yang X, Xing Y, Chen Z. 5'-triphosphate-siRNA activates RIG-I-dependent type I interferon production and enhances inhibition of hepatitis B virus replication in HepG2.2.15 cells. Eur J Pharmacol. 2013;721:86-95.

18. Jun W, Zhong JM, Min J, et al. Hepatitis B virus suppresses toll-like receptor-mediated innate immune responses in murine parenchymal and nonparenchymal liver cells. Hepatology. 2009;49:1132-40.

19. Yang $\mathrm{H}$, Westland C, Xiong S, Delaney W. In vitro antiviral susceptibility of full-length clinical hepatitis B virus isolates cloned with a novel expression vector. Antiviral Res. 2004;61:27-36.

20. Meng Z, Xu Y, Wu J, Tian Y, Kemper T, Bleekmann B, Roggendorf M, Yang D, Lu M. Inhibition of hepatitis B virus gene expression and replication by endoribonuclease-prepared siRNA. J Virol Methods. 2008;150:27-33.

21. Wu J, Meng Z, Jiang M, Zhang E, Trippler M, Broering R, Bucchi A, Krux F, Dittmer U, Yang D, et al. Toll-like receptor-induced innate immune responses in non-parenchymal liver cells are cell type-specific. Immunology. 2010;129:363-74.

22. Wooddell Cl, Rozema DB, Hossbach M, John M, Hamilton HL, Chu Q, Hegge JO, Klein JJ, Wakefield DH, Oropeza CE, et al. Hepatocyte-targeted RNAi therapeutics for the treatment of chronic hepatitis B virus infection. Mol Ther. 2013;21:973-85.

23. Wooddell Cl, Yuen MF, Chan HL, Gish RG, Locarnini SA, Chavez D, Ferrari C, Given BD, Hamilton J, Kanner SB, et al. RNAi-based treatment of chronically infected patients and chimpanzees reveals that integrated hepatitis B virus DNA is a source of HBsAg. Sci Transl Med. 2017;9:eaan0241.

24. Smolders EJ, Burger DM, Feld JJ, Kiser JJ. Review article: clinical pharmacology of current and investigational hepatitis B virus therapies. Aliment Pharmacol Ther. 2020;51:231-43. 
25. Hensel KO, Cantner F, Bangert F, Wirth S, Postberg J. Episomal HBV persistence within transcribed host nuclear chromatin compartments involves HBx. Epigenetics Chromatin. 2018;11:34.

26. Forsbach A, Nemorin JG, Montino C, Muller C, Samulowitz U, Vicari AP Jurk M, Mutwiri GK, Krieg AM, Lipford GB, Vollmer J. Identification of RNA sequence motifs stimulating sequence-specific TLR8-dependent immune responses. J Immunol. 2008:180:3729-38.

27. Judge AD, Sood V, Shaw JR, Fang D, McClintock K, MacLachlan I. Sequence-dependent stimulation of the mammalian innate immune response by synthetic siRNA. Nat Biotechnol. 2005;23:457-62.
28. Gantier MP, Tong S, Behlke MA, Irving AT, Lappas M, Nilsson UW, Latz E, McMillan NAJ, Williams BRG. Rational Design of Immunostimulatory siRNAs. Mol Ther. 2010:18:785-95.

\section{Publisher's Note}

Springer Nature remains neutral with regard to jurisdictional claims in published maps and institutional affiliations.
Ready to submit your research? Choose BMC and benefit from:

- fast, convenient online submission

- thorough peer review by experienced researchers in your field

- rapid publication on acceptance

- support for research data, including large and complex data types

- gold Open Access which fosters wider collaboration and increased citations

- maximum visibility for your research: over $100 \mathrm{M}$ website views per year

At BMC, research is always in progress.

Learn more biomedcentral.com/submissions 